\title{
Brain Functional Network in Alzheimer's Disease: Diagnostic Markers for Diagnosis and Monitoring
}

\author{
Guido Rodriguez, Dario Arnaldi, and Agnese Picco \\ Department of Neurosciences, Ophthalmology, and Genetics, Clinical Neurophysiology Unit, University of Genoa, De Toni street 5, \\ 16132 Genoa, Italy \\ Correspondence should be addressed to Dario Arnaldi, dario.arnaldi@gmail.com
}

Received 14 December 2010; Revised 8 March 2011; Accepted 22 March 2011

Academic Editor: Giuseppe Curcio

Copyright ( $\odot 2011$ Guido Rodriguez et al. This is an open access article distributed under the Creative Commons Attribution License, which permits unrestricted use, distribution, and reproduction in any medium, provided the original work is properly cited.

\begin{abstract}
Alzheimer's disease $(\mathrm{AD})$ is the most common type of dementia that is clinically characterized by the presence of memory impairment and later by impairment in other cognitive domains. The clinical diagnosis is based on interviews with the patient and his/her relatives and on neuropsychological assessment, which are also used to monitor cognitive decline over time. Several biomarkers have been proposed for detecting $\mathrm{AD}$ in its earliest stages, that is, in the predementia stage. In an attempt to find noninvasive biomarkers, researchers have investigated the feasibility of neuroimaging tools, such as MR, SPECT, and FDG-PET imaging, as well as neurophysiological measurements using EEG. In this paper, we investigate the brain functional networks in AD, focusing on main neurophysiological techniques, integrating with most relevant functional brain imaging findings.
\end{abstract}

\section{Introduction}

Amnesic mild cognitive impairment (MCI) is characterized by memory impairment, either associated or not with mild deficit in other cognitive domains whereas the function of daily living is essentially preserved [1-3]. Annual conversion rate from normality to dementia of Alzheimer's type (Alzheimer's disease, AD) ranges between $0.2 \%$ and $4 \%[3,4]$ whereas that from MCI to $\mathrm{AD}$ is between $6 \%$ and $25 \%$ $[3,5]$. It is an open issue with important clinical implications whether or not MCI is essentially a prodromic stage of $\mathrm{AD}$ [3].

Although clinical manifestations of cognitive dysfunction and impairments of activities of daily living are the current standard measures for the diagnosis of $\mathrm{AD}$, biomarkers are receiving increasing attention in research centers as possible early diagnostic surrogate measures of the ongoing pathology [6]. Not surprisingly, there is already a growing literature of biomarkers associated with the transition of MCI to AD [7$14]$.

Connectivity plays a critical role in mediating cognitive function. The breakdown of connectivity, both in the functional and structural system domain, plays a major role in the onset of $\mathrm{AD}$ symptoms. Thus, a failure of the regions of a network to interact at a high level of coordination may underpin the cognitive disorders which are present in AD. The failure of network function may be due to interaction failure among the regions of a network, which is denoted as the disconnection hypothesis [15]. The breakdown is thought to be due to chronically progressive $\mathrm{AD}$ neuropathology with underlying molecular mechanisms leading downstream to neuronal and synaptic dysfunction and ultimately to neuronal loss. Such AD-characteristic structural and functional changes are hypothesized to reflect, at least partially, the progressive impairment of fiber tract connectivity and integrity [16-18], suggesting that disconnection in $\mathrm{AD}$ is evident at both the functional and structural level.

Advances in electroencephalographic (EEG) signal analysis permit relatively precise localization of brain neural sources and the ability to track their hierarchical connectivity in sustaining a given function. This information can be integrated with structural and functional imaging provided by fluorodeoxyglucose (FDG) positron emission tomography (PET), perfusion single-photon emission computed tomography (SPECT), and functional magnetic resonance imaging (fMRI). Such integrated measures can index patterns of 
neural activation responsible for sensory perception, attention, memory, movement, and higher mental operations including language and thought, since electromagnetic signals change in parallel over time and task, and can be impaired directly during such activity [19].

Actually, in the new guidelines for the AD diagnosis [20], EEG is not mentioned as a diagnostic measurement, instead of giving greater emphasis on MRI, cerebrospinal fluid (CSF), PET, and genetic findings.

The associations between brain pathology and indices of functional and structural connectivity may help our understanding of the role of connectivity in brain function [15].

The aim of this review is to investigate the brain functional network in AD focusing on main neurophysiological techniques and integrating the results with functional brain imaging findings. We will mainly review studies using EEG data to investigate functional networks; moreover, some very recent studies utilizing PET and SPECT to investigate functional brain imaging of $\mathrm{AD}$-related pathology are reported.

\section{Functional Network}

2.1. EEG in Normal Aging. Studies in normal elderly individuals have consistently showed that healthy ageing is not associated with substantial EEG changes, which instead are caused by pathological conditions. Usually, the EEG signal is elaborated (quantitative EEG-qEEG) performing a fast Fourier transform (FFT) in order to estimate the power density of selected EEG frequency band, providing a power spectrum and high-density spatial EEG mapping of each frequency band.

A tendency toward a slower alpha rhythm has been reported in the elderly subjects, but it is poorly significant in comparison to normal adults. In fact, the normal alpha frequency is higher than $8 \mathrm{~Hz}$ also in the elderly. A qEEG study of age-related changes during cognitive tasks revealed no conclusive differences between the young and the elderly [21]. Therefore, it should be taken in mind that an abnormal EEG in aged people should prompt further investigation to disclose brain pathology, since normal aging per se is not associated with significant EEG alterations.

To make this point clear, it is noteworthy that slow waves over the temporal areas (mainly of the left hemisphere) are occasionally seen in the EEG of normal elderly subjects. The main features of these "nonpathological" slow waves are that they do not disrupt background activity, they are not associated with a substantial asymmetry of the alpha rhythm, their morphology is usually rounded, and their voltage is usually greater than $60-70 \mu \mathrm{V}$. Moreover, they are attenuated by mental activity and eye opening, and their prevalence is increased by drowsiness and hyperventilation. Finally, they occur sporadically as single waves or in pairs, not in longer rhythmic trains.

2.2. The Role of EEG in AD. Although EEG is the only clinical diagnostic instrument directly reflecting cortical neuronal functioning, the genesis of surface EEG rhythms is still the object of current investigation and partly not understood. The biological complexity of the brain modular function and the physical "sum" effect of different brain electrical fields on surface EEG recordings make the understanding of EEG components a very difficult task.

In general, EEG changes are well related to cognitive dysfunction in AD. Moreover, cognitive impairment is associated with a reduction or loss of EEG reactivity in $\mathrm{AD}$ [22]. Normal alpha was shown to be suppressed during eye opening in $\mathrm{AD}$ patients with significantly higher WAIS performance IQ scores whereas in AD patients with irregular alpha it does not or only weakly change during eye opening [23].

The most frequent findings are the power reduction of beta activity and alpha rhythm, the power increase of slow activities in the theta bands in milder dementias, and of delta activities in more severe dementia. Both intrahemispheric and interhemispheric coherence of fast and alpha EEG activities is reduced in neurodegenerative diseases causing dementia, thus suggesting a reduction of neural connections. On the contrary, coherence in delta and theta bands have been reported to be increased in $\mathrm{AD}$, but this data is not agreed upon by all researchers [24]. The alpha (or background activity) also suffers from the slowing-down of its frequency, often till its peak falls below the $8-8.5 \mathrm{~Hz}$. This phenomenon can happen together with a true increase of the theta power.

According to the "transition" hypothesis that considers MCI as a "reservoir" of patients possibly developing dementia, mainly of the $\mathrm{AD}$ type, EEG studies have tried to highlight early changes. Considered altogether, it is difficult to identify MCI patients from normal controls, but emerging data is consistent with the hypothesis that those who will convert to dementia already show similar EEG changes as early $\mathrm{AD}$ patients $[7,8,13,25]$. Moreover qEEG features could predict longitudinal cognitive decline in normal elderly with subjective complaints, with an overall predictive accuracy of $90 \%$ [26].

It should be taken in mind that EEG measures electrical field variations, and a number of clinical conditions can disturb the normal electrical field of the brain. For instance, electrolyte changes may alter the appearance and time variation of the brain-generated electrical fields, and medications can slow the posterior dominant rhythm. Moreover, in assessing the frequency of the alpha rhythm, alerting manoeuvres are essential in order to ensure that the patient is not drowsy. Hence, a large number of conditions cause the EEG to appear abnormal. In EEG practice, the clinician has to rely to a large extent on the clinical history and the neurological examination findings to make a clinically meaningful conclusion.

In summary, a shift-to-the-left of background activity and the increase of theta power are the earliest and more robust features of $\mathrm{AD}$. When the disease progresses to its moderate stage, theta activities increase further and delta activities appear. In the most severe stages, delta and theta activities increase again while the background activity cannot be longer recognized. These increasing EEG changes according to severity of AD have been highlighted by a study based 


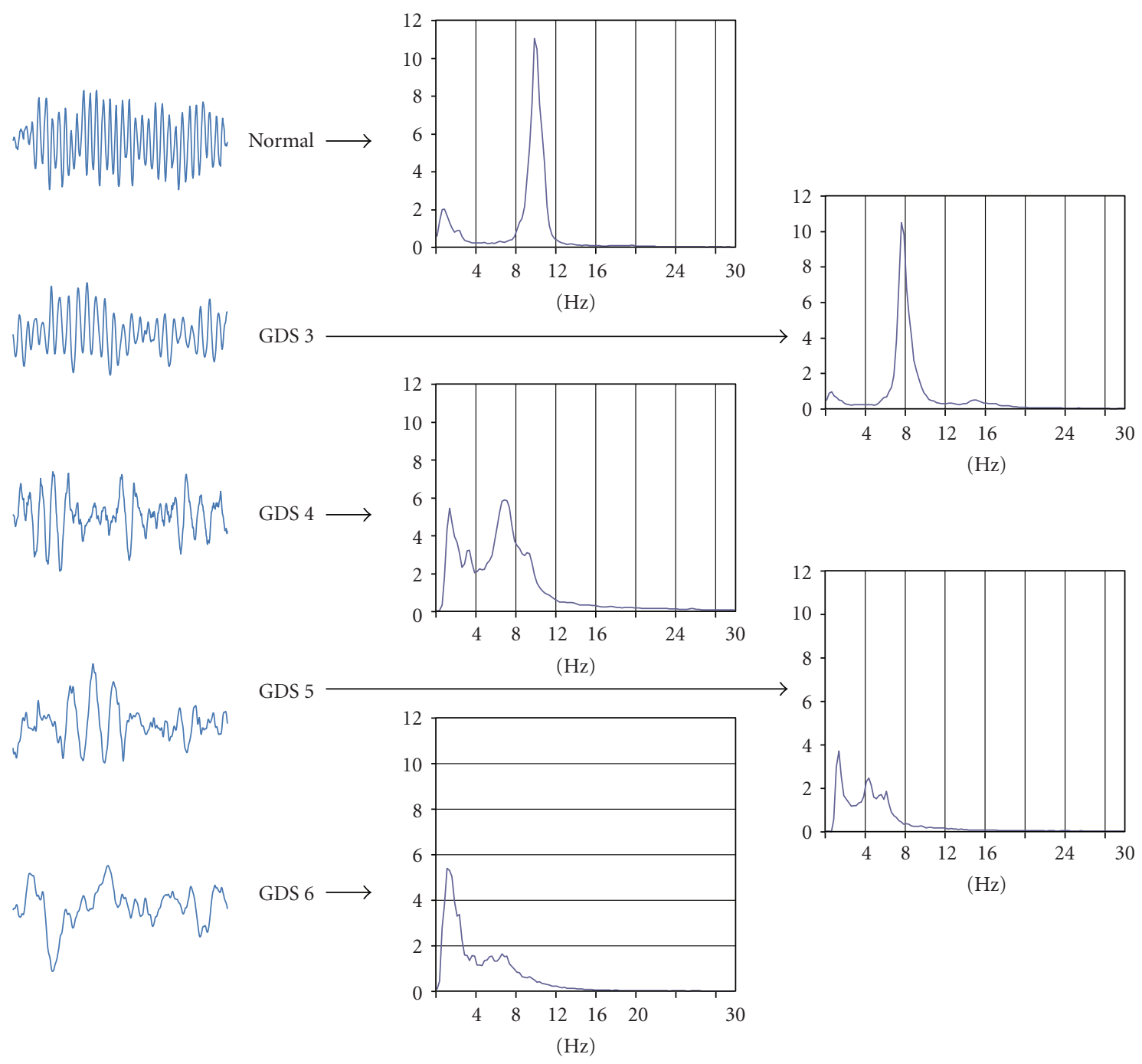

FIGURE 1: Sample of visual EEG and EEG spectrum on 4 clinical classes of severity (Global Deterioration Scale: GDS, from 3 to 6; for more details see text). EEG frequency bands ( $X$-axis) and percent value of each band ( $Y$-axis) are shown.

on 4 clinical classes of severity (GDS, from 3 to 6, Figures 1 and 2) [27].

2.3. Pathophysiology of EEG Changes in AD. With this basis, the understanding of pathophysiology of EEG changes in $\mathrm{AD}$ is even more complex and just some general concepts can be commented. Scalp alpha rhythms $(8-13 \mathrm{~Hz})$ mainly result from sequences of inhibitory (IPSP) and excitatory (EPSP) postsynaptic potentials at the dendrites of cortical pyramidal neurons. These potentials depend mainly on the influence of near and distant cortical modules [28], as well as on the interactions of excitatory corticothalamocortical relay fibres and inhibitory thalamic reticular fibres [29, 30]. Cholinergic and glutamatergic synapses are especially involved in the genesis of these potentials. In Alzheimer's disease (AD), characterized by an early cholinergic (and possibly glutamatergic) deficit, this may produce a slowingdown of alpha rhythm and a reduction up to disappearance of alpha rhythm in the severe stages.
Theta rhythms are usually not appreciated in normal awakening EEG. However, a theta power increase is observed over the frontal and temporal areas during learning and memory tasks. The theta rhythms that are recorded during these tasks are thought to be produced by the activation of septal-hippocampal system. Hippocampus has a cholinergic innervation originating from basal forebrain, the medial septum, and the vertical limb of the diagonal band of Broca. Populations of GABAergic and glutamatergic neurons have also been described in several basal forebrain structures. The synchronized depolarization of hippocampal neurons produces field potentials that have a main frequency of $3-12 \mathrm{~Hz}$ and are usually known as hippocampal theta rhythm [31]. A cholinergic-glutamatergic hypothesis of $\mathrm{AD}$, in which most symptoms may be explained by cholinergic-glutamatergic deficits, has been advanced. Neuronal injury/loss may include an excitotoxic component that possibly contributes to the early cholinergic deficit. This excitotoxic component may occur, at least in part, at the septal level where somas 


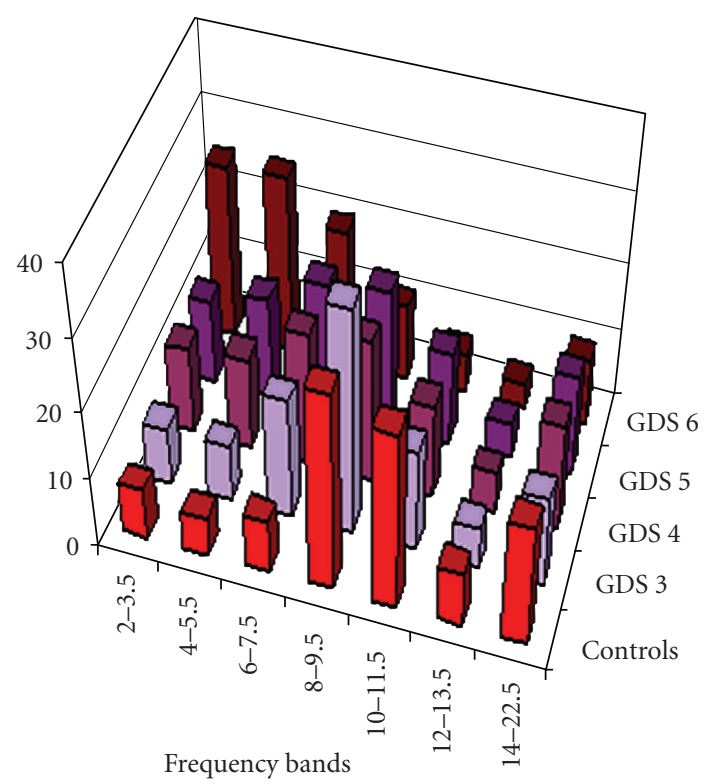

FIGURE 2: Histogram showing the relationship between 7 EEG frequency bands $(2-3.5 ; 4-5.5 ; 6-7.5 ; 8-9.5 ; 10-11.5 ; 12-13.5 ; 14-$ $22.5 \mathrm{~Hz}$ ) and disease's severity (normal controls and 4 clinical classes of severity; GDS 3 to 6 ).

of cholinergic neurons are found. This insult may modify septal networks and contribute to the abnormal information processing observed in $\mathrm{AD}$ brain, including its hyperexcitability states. According to this theory, the increased theta production in $\mathrm{AD}$ would derive from hyperexcitability of the septal-hippocampal system [32].

By means of observations in head injury patients, it has been suggested that delays in corticocortical fiber propagation may play a global role in determining human EEG frequencies, increasing the amount of delta activity [33]. Increased T2 relaxation times in cortical gray matter and white matter were correlated with a shift in relative EEG power to lower frequencies in the delta range (delta activity: $1-4 \mathrm{~Hz}$ ) and reduced cognitive performance. Generally, these data are consistent with the idea that head injury somehow damages the ability of brains to form local cell assemblies within the global synaptic action field environment.

The increment of delta oscillations in mild cognitive impairment $(\mathrm{MCI})$ and $\mathrm{AD}$ subjects might be related to loss of hippocampal and posterior cortical neurons, which are impinged by cholinergic inputs. Indeed, it has been demonstrated that early degeneration in mesial temporal cortex of $\mathrm{AD}$ subjects can affect functional connectivity between hippocampal formation and temporoparietal cortex [34]. Furthermore, a bilateral reduction of gray matter volume in the hippocampal formation and entorhinal cortex of $\mathrm{AD}$ subjects was correlated with an increment of delta rhythms in posterior cortex $[9,35]$.

\section{Functional Brain Imaging}

Historically, morphological imaging became easily a reliable diagnostic procedure for several brain disease, like neoplastic or cerebrovascular injuries, and at least for degenerative disease. This did not happen for functional neuroimaging, either metabolic or perfusional. This has actually slowed down these biomarkers introduction into dementia diagnostic criteria.

Recently, Dubois et al. proposed to revise the NINCDSADRDA criteria for the diagnosis of AD [20]. A specific pattern on functional neuroimaging with FDG-PET has been proposed as one of the supportive features in the diagnosis of probable $\mathrm{AD}$, specifically in terms of reduced glucose metabolism in bilateral temporal parietal regions. In fact, a reduction of glucose metabolism as seen on PET in bilateral temporal parietal regions and in the posterior cingulate is the most commonly described diagnostic criterion for AD [36].

The newly proposed diagnostic criteria for $\mathrm{AD}$ entails a two-step diagnostic process, first identifying dementia syndrome (lack of episodic memory and other cognitive impairment) and then applying criteria based on the AD phenotype (presence of plaque and neurofibrillary tangles) [20]. As a matter of fact, this does not allow diagnosis in life. Furthermore, the pathogenetic role of amyloid deposition in $\mathrm{AD}$ patients is still unclear, highlighting the necessity of another diagnostic path [37-40]. In summary, the authors propose that the term "Alzheimer's disease" should refer only to the in vivo clinicobiological expression of the disease. Obviously, prospective studies with postmortem verification are needed to validate this new proposal. Actually, metabolic changes (as identified by FDG-PET) associated with neocortical dysfunction are detectable before atrophy appears [41]. Moreover, metabolism reductions exceeded volume losses in MCI [42], and in presymptomatic early-onset familial AD [43]. Actually, a pattern of parietotemporal metabolic reductions in MCI and AD, and frontal metabolic reductions later in the disease, has been established through the last decades of research [44-46] and has recently been confirmed in ADNI PET data [47]. The usefulness of FDG-PET could be highlighted also in detecting prodromal AD showing metabolic reductions in the anterior cingulate, posterior cingulate, and temporal, parietal, and medial temporal cortices [48-50].

Finally, several compounds have been developed for the imaging of amyloid for PET and SPECT. The rapid development of different compounds suitable for the visualising of amyloid during the past 10 years has led to the first promising in vivo studies of the amyloid ligands PIB (N-methyl2-(4L'-methyl aminophenyl)-6-hydroxybenzothiazole) [51] and FDDNP (2-(1-[6-[(2-[18F]fluoroethyl](methyl)amino]-2-naphthyl] ethylidene)malononitrile) [52]; the latter compound also seems to label neurofibrillary tangles in patients with AD. Furthermore, both compounds have shown a pattern of increased radioligand retention in patients with AD compared with control individuals that is consistent with AD pathology [52-54]. Accumulation of amyloid, however, has also been reported in cognitively intact older people [3740]. In a recent paper, Oh et al. using PET imaging with the PIB compound, structural MRI, and cognitive measures identify two brain networks in which the degree of gray matter volume fluctuates in a similar manner: a frontal network and a posterior network [39]. The authors suggested that $\beta$-amyloid deposition in older people without dementia 
may influence a wide structural network, although it is not clear whether people with higher $\beta$-amyloid deposition will progress to $\mathrm{AD}$.

Because SPECT is more widely available and cheaper than PET, it has received much attention as an alternative to PET. However, at present, the technique is not included in the criteria proposed by Dubois et al. [20] as the diagnostic accuracy estimates for this modality generally fall below the requisite $80 \%$ levels specified by the Reagan Biomarker Working Group [55].

\section{Neurophysiological Evaluation of AD}

In a clinical context, some firm points can be made concerning EEG in the evaluation of AD. In a more strict sense, the main applications of EEG should be as a different diagnostic tool between dementia and other conditions characterized by peculiar EEG pattern such as Creutzfeldt-Jakob disease (CJD), toxic-metabolic encephalopathy, or in case of pseudodepressive dementia [56]. In a broader sense, EEG can be useful to stage the severity of dementia on a pathophysiological basis, and, in AD, gives useful information for prognostic purposes [57]. Actually, all patients with moderate to severe $\mathrm{AD}$ could exhibit abnormal EEGs. When a substantial part of the dominant rhythm falls within the range of theta band physicians should be encouraged to perform qEEG. This, in order to identify the so-called transition frequency between dominant and theta activity, as suggested by Klimesh [58]. Moreover, qEEG is a highly sensitive method to evaluate the biological effect of drugs [59,60].

Cortical sources of scalp EEG rhythms have been successfully evaluated in AD patients by single dipole sources deeply located into a spherical brain model [61]. Single dipole sources of alpha or beta rhythms are located more anteriorly as a function of $\mathrm{AD}$ severity. Such "anteriorization" of the dipole source is observed in $\mathrm{AD}$ patients not only with respect to normal subjects but also with respect to subjects with MCI $[8,61]$. Notably, the location of the dipole sources correlates with the reduction of $\mathrm{rCBF}$ in anteroposterior and laterolateral brain axes [62]. By applying the LORETA technique, which elaborates solutions to compute the cortical sources of EEG activities, several multicenter studies have been performed in recent years in $\mathrm{AD}$ as well as in $\mathrm{MCI}$, gaining substantial information [7-9, 63].

Even if not usually used in clinical practice, other neurophysiological measurements could be performed in the evaluation of AD. Event-related potentials (ERP) may reflect cognitive decline in the longitudinal followup of MCI [64] and AD patients [65], and ERP and MRI data fusion could improve diagnostic accuracy of early AD [66]. Moreover, transcranial magnetic stimulation (TMS), especially combined with EEG, may provide useful information about the degree and progression of AD [67-69].

However, it is obviously important to combine multiple biomarkers in order to obtain complementary information to be used in clinical AD diagnosis practice. This kind of investigation has been recently performed [41, 70-72] confirming that each biomarker (including EEG, PET, SPECT,
MRI, apolipoprotein E risk gene (ApoE4), cerebrospinal fluid (CSF), and neuropsychological tests) does carry complementary information, and the simple combination of classifiers trained on these different modalities can improve the diagnostic performance. Indeed, ApoE2 has been suggested as having a protective effect and delaying the age of onset of $\mathrm{AD}[73,74]$.

qEEG has been analysed together with other measures of brain function. For instance, qEEG was analysed together with regional cerebral blood flow ( $\mathrm{rCBF}$ ) quantitative measurements in order to investigate the correlation between EEG activities and hypoperfusion and to assess the diagnostic accuracy of the two methods used alone or in combination. In a study on $42 \mathrm{AD}$ patients and 18 healthy controls [75], $\mathrm{rCBF}$ and $\mathrm{qEEG}$ were correlated with one another, suggesting that these measurements used together are reasonably accurate in differentiating AD from healthy aging. Another qEEG-SPECT (semiquantitative Tc-99 HMPAO technique) correlative study on $42 \mathrm{AD}$ patients underlined that bilateral hippocampal rCBF was the perfusional index best correlated with the MMSE as well as being significantly correlated to qEEG [76] (Figures 3, 4, and 5).

A very interesting application of qEEG measures tried to evaluate their prognostic meaning in AD. In a preliminary study on $31 \mathrm{AD}$ patients, right delta relative power predicted both the loss of activities of daily living (ADL) and death whereas right theta relative power predicted the onset of incontinence [77]. A confirmation came from an extended group of 72 patients. Because patients were in different stages of the disease, the statistical analysis was performed in the entire group as well as in the subgroup of 41 patients with mild AD (scoring 3 or 4 on the GDS). In the whole group, the loss of ADL was predicted by delta relative power in either side, incontinence was predicted by alpha relative power in the right side, a borderline statistical significance was reached for death $(P<.05)$. In the subgroup of mildly demented patients, the loss of ADL was predicted by left delta relative power, incontinence by both delta and alpha relative powers in the right side, and death was not significantly predicted $(P=.08)[78]$.

Using both conventional visual analysis and qEEG, other authors found that AD patients with an abnormal EEG at an early stage had a different pattern of cognitive decline than those (matched for severity of dementia) with a normal EEG. The patients with a deteriorating EEG during the first year of followup subsequently showed a greater decline of praxic functions, a tendency to Parkinsonism and a higher risk of institutionalisation than patients with a stable EEG during the 1st year [79]. In another study, more marked EEG abnormalities were found in patients with delusions and hallucinations who also showed a more rapid cognitive decline [80]. The same authors also found that an abnormal EEG and psychosis were independent predictors of disease progression [81].

As discussed in a recent paper [8], most of the EEG studies in $\mathrm{AD}$ patients have reported a prominent decrease of coherence at the alpha band. The reduction of alpha coherence in $\mathrm{AD}$ patients has been also found to be associated with ApoE genetics risk of dementia; this alpha power reduction 

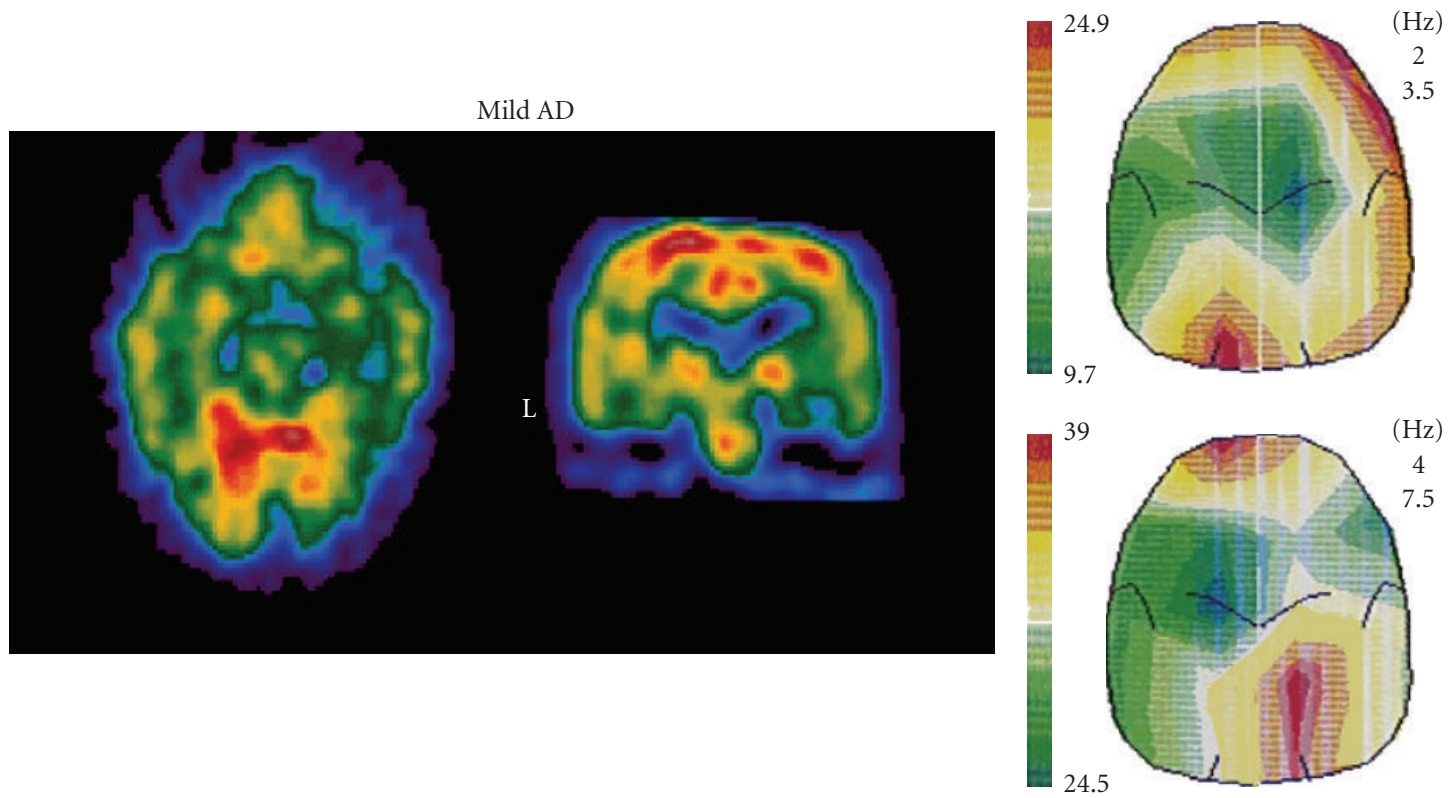

FIGURE 3: Sample of SPECT neuroimaging (Tc-99 HMPAO) and EEG brain mapping in a mild AD patient. Topographic scalp distribution of the EEG power on the 2.0 to $3.5 \mathrm{~Hz}$ frequency band (top right) and 4.0 to $7.5 \mathrm{~Hz}$ frequency band (bottom right) is shown. For more details, see text.
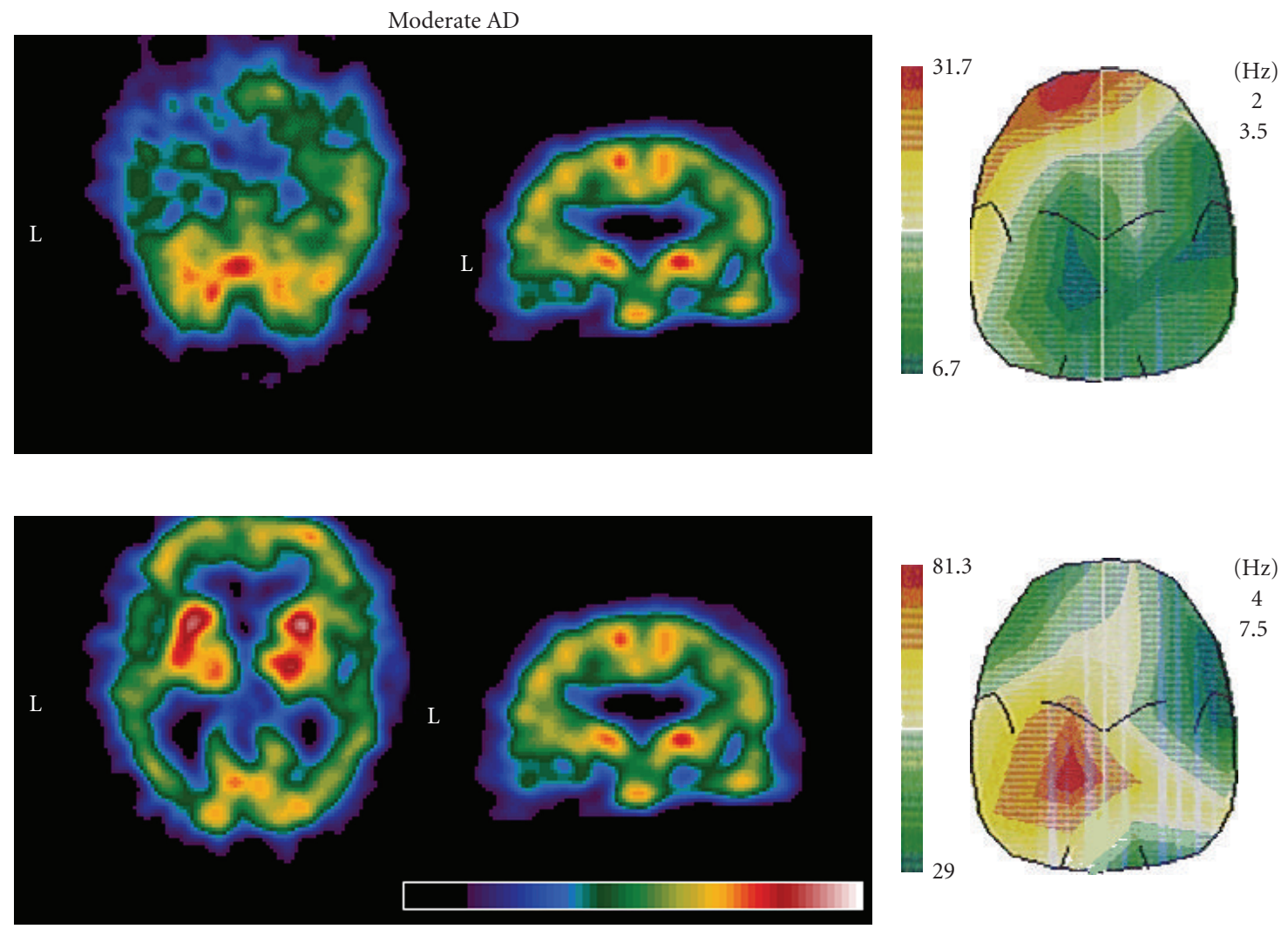

FIgURE 4: Sample of SPECT neuroimaging (Tc-99 HMPAO) and EEG brain mapping in a moderate AD patient. Topographic scalp distribution of the EEG power on the 2.0 to $3.5 \mathrm{~Hz}$ frequency band (top right) and 4.0 to $7.5 \mathrm{~Hz}$ frequency band (bottom right) is shown. For more details, see text. 

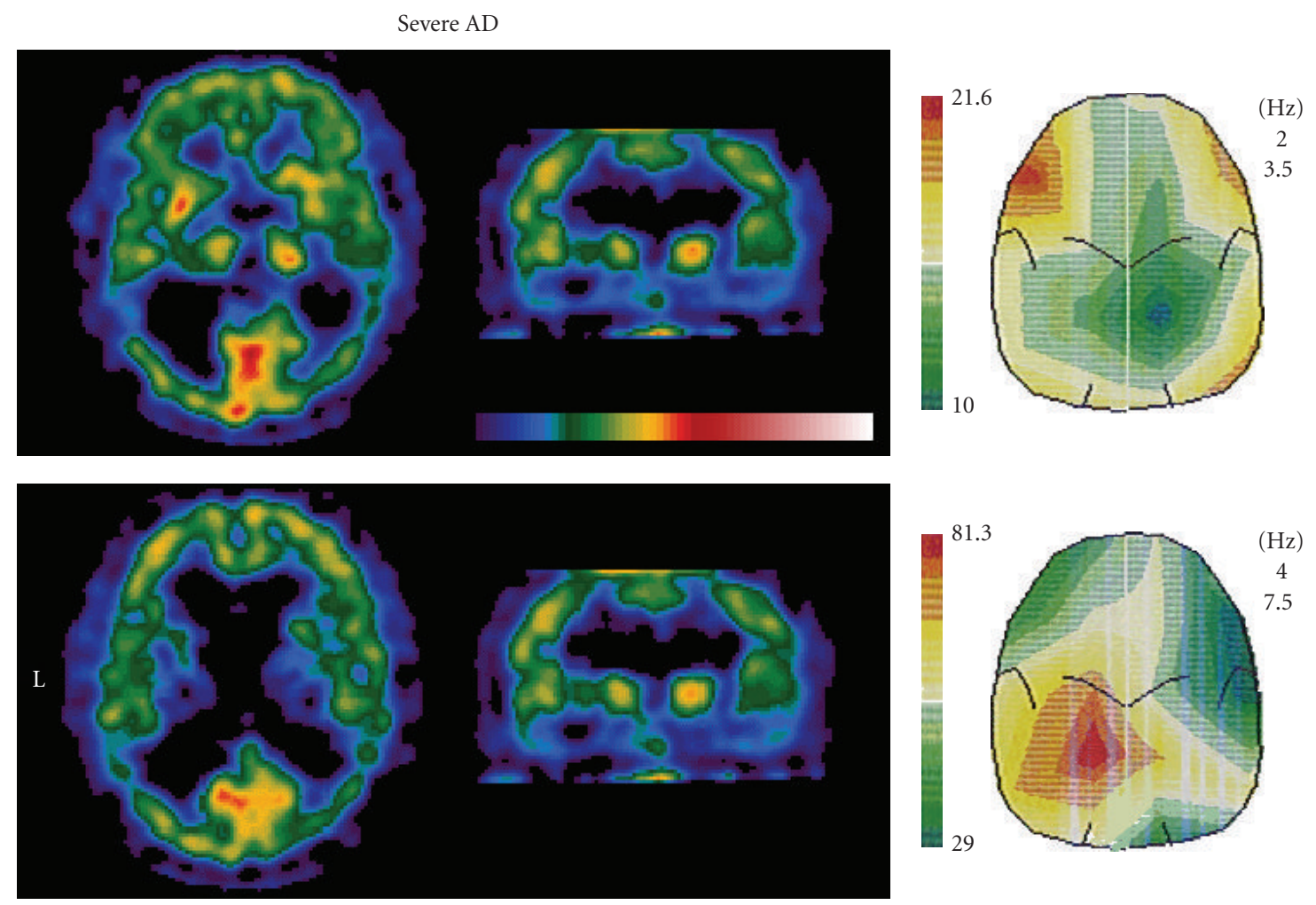

FIGURE 5: Sample of SPECT neuroimaging (Tc-99 HMPAO) and EEG brain mapping in a severe AD patient. Topographic scalp distribution of the EEG power on the 2.0 to $3.5 \mathrm{~Hz}$ frequency band (top right) and 4.0 to $7.5 \mathrm{~Hz}$ frequency band (bottom right) is shown. For more details, see text.

is supposed to be mediated by cholinergic deficit [82] Instead, coherence at the delta and theta bands has been less straightforward. Some studies have shown a decrement of slow EEG coherence in AD patients [83] whereas others have reported its increase [84]. Wada et al. [85] examined intrahemispheric coherence at rest and during photic stimulation in $10 \mathrm{AD}$ patients. In the resting EEG, patients with $\mathrm{AD}$ had significantly lower coherence than gender- and agematched healthy control subjects in the alpha-1, alpha-2, and beta-1 frequency bands. EEG analysis during photic stimulation demonstrated that the patients had significantly lower coherence, irrespective of the stimulus frequency. The changes in coherence from the resting state to the stimulus condition showed significant group differences in the region of the brain primarily involved in visual functioning. These findings suggest that patients with $\mathrm{AD}$ may have an impairment of functional connectivity in both nonstimulus and stimulus conditions. This suggests a failure of normal stimulation-related brain activation in AD. In another study, alpha coherence was decreased significantly in temporo-parieto-occipital areas in the majority of patients while significant delta coherence increase was found in a few patients between frontal and posterior regions. This was expressed to a greater extent in patients with a more severe cognitive impairment [84]. The authors speculated that their findings could reflect two different pathophysiological changes: (i) the alpha coherence decrease could be related to alterations in corticocortical connections whereas (ii) the delta coherence increase suggests lack of influence of subcortical cholinergic structures on cortical electrical activity.

Finally, the EEG correlates of biological markers have been investigated in AD. Jelic et al. [86] found a positive correlation between levels of tau protein in the cerebrospinal fluid (CSF) and delta/alpha ratio. In a subgroup with high CSF tau levels, a strong relationship between EEG alpha/theta and alpha/delta power ratios was found. No such correlation was found in healthy controls and mildly cognitively impaired individuals with elevated CSF tau levels. ApoE 4 allele is a risk factor for late-onset AD and is proposed to have an impact on cholinergic function in AD.

The qEEG of 31 patients with AD was recorded at the early stage of the disease and after a 3-year followup. Patients with $\mathrm{AD}$ were divided into several subgroups according to the number of ApoE4 alleles, with a similar clinical severity and duration of dementia. The AD patients carrying the ApoE4 alleles had more pronounced slow-wave activity than $\mathrm{AD}$ patients without the ApoE4 alleles, although the disease progression rate did not change. These differences in EEG may suggest differences in the degree of the cholinergic deficit in these subgroups [87]. 


\section{References}

[1] C. Flicker, S. H. Ferris, and B. Reisberg, "Mild cognitive impairment in the elderly: predictors of dementia," Neurology, vol. 41, no. 7, pp. 1006-1009, 1991.

[2] F. Portet, P. J. Ousset, P. J. Visser et al., "Mild cognitive impairment (MCI) in medical practice: a critical review of the concept and new diagnostic procedure. Report of the MCI Working Group of the European Consortium on Alzheimer's Disease," Journal of Neurology, Neurosurgery and Psychiatry, vol. 77, no. 6, pp. 714-718, 2006.

[3] R. C. Petersen, R. Doody, A. Kurz et al., "Current concepts in mild cognitive impairment," Archives of Neurology, vol. 58, no. 12, pp. 1985-1992, 2001.

[4] G. B. Frisoni, A. Padovani, and L. O. Wahlund, "The predementia diagnosis of Alzheimer disease," Alzheimer Disease and Associated Disorders, vol. 18, no. 2, pp. 51-53, 2004.

[5] R. C. Petersen, G. E. Smith, S. C. Waring, R. J. Ivnik, E. G. Tangalos, and E. Kokmen, "Mild cognitive impairment: clinical characterization and outcome," Archives of Neurology, vol. 56, no. 3, pp. 303-308, 1999.

[6] T. Sunderland, H. Hampel, M. Takeda, K. T. Putnam, and R. M. Cohen, "Biomarkers in the diagnosis of Alzheimer's disease: are we ready?" Journal of Geriatric Psychiatry and Neurology, vol. 19, no. 3, pp. 172-179, 2006.

[7] C. Babiloni, G. Binetti, E. Cassetta et al., "Sources of cortical rhythms change as a function of cognitive impairment in pathological aging: a multicenter study," Clinical Neurophysiology, vol. 117, no. 2, pp. 252-268, 2006.

[8] C. Babiloni, R. Ferri, G. Binetti et al., "Fronto-parietal coupling of brain rhythms in mild cognitive impairment: a multicentric EEG study," Brain Research Bulletin, vol. 69, no. 1, pp. 63-73, 2006.

[9] C. Babiloni, G. Frisoni, M. Steriade et al., "Frontal white matter volume and delta EEG sources negatively correlate in awake subjects with mild cognitive impairment and Alzheimer's disease," Clinical Neurophysiology, vol. 117, no. 5, pp. 11131129, 2006.

[10] P. M. Rossini, C. Del Percio, P. Pasqualetti et al., "Conversion from mild cognitive impairment to Alzheimer's disease is predicted by sources and coherence of brain electroencephalography rhythms," Neuroscience, vol. 143, no. 3, pp. 793-803, 2006.

[11] G. Chételat, B. Desgranges, V. De la Sayette, F. Viader, F. Eustache, and J. C. Baron, "Mild cognitive impairment: can FDG-PET predict who is to rapidly convert to Alzheimer's disease?" Neurology, vol. 60, no. 8, pp. 1374-1377, 2003.

[12] C. R. Jack Jr., M. M. Shiung, S. D. Weigand et al., "Brain atrophy rates predict subsequent clinical conversion in normal elderly and amnestic MCI," Neurology, vol. 65, no. 8, pp. 12271231, 2005.

[13] V. Jelic, S. E. Johansson, O. Almkvist et al., "Quantitative electroencephalography in mild cognitive impairment: longitudinal changes and possible prediction of Alzheimer's disease," Neurobiology of Aging, vol. 21, no. 4, pp. 533-540, 2000.

[14] E. S. C. Korf, L. O. Wahlund, P. J. Visser, and P. Scheltens, "Medial temporal lobe atrophy on MRI predicts dementia in patients with mild cognitive impairment," Neurology, vol. 63, no. 1, pp. 94-100, 2004.

[15] A. L. W. Bokde, M. Ewers, and H. Hampel, "Assessing neuronal networks: understanding Alzheimer's disease," Progress in Neurobiology, vol. 89, no. 2, pp. 125-133, 2009.
[16] K. Meguro, X. Blaizot, Y. Kondoh, C. Le Mestric, J. C. Baron, and C. Chavoix, "Neocortical and hippocampal glucose hypometabolism following neurotoxic lesions of the entorhinal and perirhinal cortices in the non-human primate as shown by PET. Implications for Alzheimer's disease," Brain, vol. 122, no. 8, pp. 1519-1531, 1999.

[17] J. H. Morrison and P. R. Hof, "Selective vulnerability of corticocortical and hippocampal circuits in aging and Alzheimer's disease," Progress in Brain Research, vol. 136, pp. 467-486, 2002.

[18] T. R. Stoub, L. DeToledo-Morrell, G. T. Stebbins, S. Leurgans, D. A. Bennett, and R. C. Shah, "Hippocampal disconnection contributes to memory dysfunction in individuals at risk for Alzheimer's disease," Proceedings of the National Academy of Sciences of the United States of America, vol. 103, no. 26, pp. 10041-10045, 2006.

[19] P. M. Rossini, S. Rossi, C. Babiloni, and J. Polich, "Clinical neurophysiology of aging brain: from normal aging to neurodegeneration," Progress in Neurobiology, vol. 83, no. 6, pp. 375-400, 2007.

[20] B. Dubois, H. H. Feldman, C. Jacova et al., "Research criteria for the diagnosis of Alzheimer's disease: revising the NINCDSADRDA criteria," Lancet Neurology, vol. 6, no. 8, pp. 734-746, 2007.

[21] M. M. Widagdo, J. M. Pierson, and R. D. Helme, "Age-related changes in qEEG during cognitive tasks," International Journal of Neuroscience, vol. 95, no. 1-2, pp. 63-75, 1998.

[22] A. Stevens and T. Kircher, "Cognitive decline unlike normal aging is associated with alterations of EEG temporo-spatial characteristics," European Archives of Psychiatry and Clinical Neuroscience, vol. 248, no. 5, pp. 259-266, 1998.

[23] P. H. Sheridan, S. Sato, N. Foster et al., "Relation of EEG alpha background to parietal lobe function in Alzheimer's disease as measured by positron emission tomography and psychometry," Neurology, vol. 38, no. 5, pp. 747-750, 1988.

[24] L. Leocani and G. Comi, "EEG coherence in pathological conditions," Journal of Clinical Neurophysiology, vol. 16, no. 6, pp. 548-555, 1999.

[25] C. Huang, L. O. Wahlund, T. Dierks, P. Julin, B. Winblad, and V. Jelic, "Discrimination of Alzheimer's disease and mild cognitive impairment by equivalent EEG sources: a crosssectional and longitudinal study," Clinical Neurophysiology, vol. 111, no. 11, pp. 1961-1967, 2000.

[26] L. S. Prichep, E. R. John, S. H. Ferris et al., "Prediction of longitudinal cognitive decline in normal elderly with subjective complaints using electrophysiological imaging," Neurobiology of Aging, vol. 27, no. 3, pp. 471-481, 2006.

[27] G. Rodriguez, F. Copello, P. Vitali, G. Perego, and F. Nobili, "EEG spectral profile to stage Alzheimer's disease," Clinical Neurophysiology, vol. 110, no. 10, pp. 1831-1837, 1999.

[28] P. L. Nunez, B. M. Wingeier, and R. B. Silberstein, "Spatial-temporal structures of human alpha rhythms: theory, microcurrent sources, multiscale measurements, and global binding of local networks," Human Brain Mapping, vol. 13, no. 3, pp. 125-164, 2001.

[29] F. H. Lopes Da Silva, J. E. Vos, J. Mooibroek, and A. Van Rotterdam, "Relative contributions of intracortical and thalamo-cortical processes in the generation of alpha rhythms, revealed by partial coherence analysis," Electroencephalography and Clinical Neurophysiology, vol. 50, no. 5-6, pp. 449-456, 1980.

[30] P. Suffczynski, S. Kalitzin, G. Pfurtscheller, and F. H. Lopes Da Silva, "Computational model of thalamo-cortical networks: 
dynamical control of alpha rhythms in relation to focal attention," International Journal of Psychophysiology, vol. 43, no. 1, pp. 25-40, 2001.

[31] B. H. Bland and L. V. Colom, "Extrinsic and intrinsic properties underlying oscillation and synchrony in limbic cortex," Progress in Neurobiology, vol. 41, no. 2, pp. 157-208, 1993.

[32] L. V. Colom, "Septal networks: relevance to theta rhythm, epilepsy and Alzheimer's disease," Journal of Neurochemistry, vol. 96, no. 3, pp. 609-623, 2006.

[33] R. W. Thatcher, C. Biver, R. McAlaster, and A. Salazar, "Biophysical linkage between MRI and EEG coherence in closed head injury," NeuroImage, vol. 8, no. 4, pp. 307-326, 1998.

[34] R. J. Killiany, M. B. Moss, M. S. Albert, T. Sandor, J. Tieman, and F. Jolesz, "Temporal lobe regions on magnetic resonance imaging identify patients with early Alzheimer's disease," Archives of Neurology, vol. 50, no. 9, pp. 949-954, 1993.

[35] A. Fernández, J. Arrazola, F. Maestú et al., "Correlations of hippocampal atrophy and focal low-frequency magnetic activity in Alzheimer disease: volumetric MR imagingMagnetoencephalographic study," American Journal of Neuroradiology, vol. 24, no. 3, pp. 481-487, 2003.

[36] R. E. Coleman, "Positron emission tomography diagnosis of Alzheimer's disease," Neuroimaging Clinics of North America, vol. 15, no. 4, pp. 837-846, 2005.

[37] H. J. Aizenstein, R. D. Nebes, J. A. Saxton et al., "Frequent amyloid deposition without significant cognitive impairment among the elderly," Archives of Neurology, vol. 65, no. 11, pp. 1509-1517, 2008

[38] M. A. Mintun, G. N. Larossa, Y. I. Sheline et al., "[11C]PIB in a nondemented population: potential antecedent marker of Alzheimer disease," Neurology, vol. 67, no. 3, pp. 446-452, 2006.

[39] H. Oh, E. C. Mormino, C. Madison, A. Hayenga, A. Smiljic, and W. J. Jagust, " $\beta$-Amyloid affects frontal and posterior brain networks in normal aging," NeuroImage, vol. 54, no. 3, pp. 1887-1895, 2011.

[40] C. C. Rowe, S. Ng, U. Ackermann et al., "Imaging $\beta$-amyloid burden in aging and dementia," Neurology, vol. 68, no. 20, pp. 1718-1725, 2007.

[41] K. B. Walhovd, A. M. Fjell, J. Brewer et al., "Combining MR imaging, positron-emission tomography, and CSF biomarkers in the diagnosis and prognosis of Alzheimer disease," American Journal of Neuroradiology, vol. 31, no. 2, pp. 347-354, 2010.

[42] S. De Santi, M. J. De Leon, H. Rusinek et al., "Hippocampal formation glucose metabolism and volume losses in MCI and AD," Neurobiology of Aging, vol. 22, no. 4, pp. 529-539, 2001.

[43] L. Mosconi, S. Sorbi, M. J. De Leon et al., "Hypometabolism exceeds atrophy in presymptomatic early-onset familial Alzheimer's disease," Journal of Nuclear Medicine, vol. 47, no. 11, pp. 1778-1786, 2006.

[44] L. Mosconi, M. Brys, L. Glodzik-Sobanska, S. De Santi, H. Rusinek, and M. J. de Leon, "Early detection of Alzheimer's disease using neuroimaging," Experimental Gerontology, vol. 42, no. 1-2, pp. 129-138, 2007.

[45] L. Mosconi, W. H. Tsui, K. Herholz et al., "Multicenter standardized F-FDG PET diagnosis of mild cognitive impairment, Alzheimer's disease, and other dementias," Journal of Nuclear Medicine, vol. 49, no. 3, pp. 390-398, 2008.

[46] M. J. De Leon, S. H. Ferris, and A. E. George, "Computed tomography and positron emission transaxial tomography evaluations of normal aging and Alzheimer's disease," Journal of Cerebral Blood Flow and Metabolism, vol. 3, no. 3, pp. 391394, 1983.

[47] J. B. S. Langbaum, K. Chen, W. Lee et al., "Categorical and correlational analyses of baseline fluorodeoxyglucose positron emission tomography images from the Alzheimer's Disease Neuroimaging Initiative (ADNI)," NeuroImage, vol. 45, no. 4, pp. 1107-1116, 2009.

[48] S. Minoshima, B. Giordani, S. Berent, K. A. Frey, N. L. Foster, and D. E. Kuhl, "Metabolic reduction in the posterior cingulate cortex in very early Alzheimer's disease," Annals of Neurology, vol. 42, no. 1, pp. 85-94, 1997.

[49] A. Drzezga, N. Lautenschlager, H. Siebner et al., "Cerebral metabolic changes accompanying conversion of mild cognitive impairment into alzheimer's disease: a PET follow-up study," European Journal of Nuclear Medicine and Molecular Imaging, vol. 30, no. 8, pp. 1104-1113, 2003.

[50] L. Mosconi, D. Perani, S. Sorbi et al., "MCI conversion to dementia and the APOE genotype: a prediction study with FDG-PET," Neurology, vol. 63, no. 12, pp. 2332-2340, 2004.

[51] S. D. Styren, R. L. Hamilton, G. C. Styren, and W. E. Klunk, "X-34, a fluorescent derivative of Congo red: a novel histochemical stain for Alzheimer's disease pathology," Journal of Histochemistry and Cytochemistry, vol. 48, no. 9, pp. 12231232, 2000.

[52] K. Shoghi-Jadid, G. W. Small, E. D. Agdeppa et al., "Localization of neurofibrillary tangles and beta-amyloid plaques in the brains of living patients with alzheimer disease," American Journal of Geriatric Psychiatry, vol. 10, no. 1, pp. 24-35, 2002.

[53] W. E. Klunk, H. Engler, A. Nordberg et al., "Imaging brain amyloid in Alzheimer's disease with Pittsburgh compound-B," Annals of Neurology, vol. 55, no. 3, pp. 306-319, 2004.

[54] N. M. Kemppainen, S. Aalto, I. A. Wilson et al., "Voxel-based analysis of PET amyloid ligand [11C]PIB uptake in Alzheimer disease," Neurology, vol. 67, no. 9, pp. 1575-1580, 2006.

[55] P. Davies, J. Resnick, B. Resnick et al., "Consensus report of the working group on: "Molecular and biochemical markers of Alzheimer's disease"," Neurobiology of Aging, vol. 19, no. 2, pp. 109-116, 1998.

[56] S. J. M. Smith, "EEG in neurological conditions other than epilepsy: when does it help, what does it add?" Neurology in Practice, vol. 76, no. 2, pp. ii8-ii12, 2005.

[57] D. Adamis, S. Sahu, and A. Treloar, "The utility of EEG in dementia: a clinical perspective," International Journal of Geriatric Psychiatry, vol. 20, no. 11, pp. 1038-1045, 2005.

[58] W. Klimesch, "EEG alpha and theta oscillations reflect cognitive and memory performance: a review and analysis," Brain Research Reviews, vol. 29, no. 2-3, pp. 169-195, 1999.

[59] K. L. Coburn, E. C. Lauterbach, N. N. Boutros, K. J. Black, D. B. Arciniegas, and C. E. Coffey, "The value of quantitative electroencephalography in clinical psychiatry: a report by the Committee on Research of the American Neuropsychiatric Association," Journal of Neuropsychiatry and Clinical Neurosciences, vol. 18, no. 4, pp. 460-500, 2006.

[60] J. R. Hughes and E. R. John, "Conventional and quantitative electroencephalography in psychiatry," Journal of Neuropsychiatry and Clinical Neurosciences, vol. 11, no. 2, pp. 190-208, 1999.

[61] T. Dierks, R. Ihl, L. Frolich, and K. Maurer, "Dementia of the Alzheimer type: effects on the spontaneous EEG described by dipole sources," Psychiatry Research, vol. 50, no. 3, pp. 151162, 1993.

[62] T. Dierks, V. Jelic, R. D. Pascual-Marqui et al., "Spatial pattern of cerebral glucose metabolism (PET) correlates with 
localization of intracerebral EEG-generators in Alzheimer's disease," Clinical Neurophysiology, vol. 111, no. 10, pp. 18171824, 2000.

[63] C. Babiloni, G. Binetti, E. Cassetta et al., "Mapping distributed sources of cortical rhythms in mild Alzheimer's disease. A multicentric EEG study," NeuroImage, vol. 22, no. 1, pp. 5767, 2004.

[64] V. T. Papaliagkas, V. K. Kimiskidis, M. N. Tsolaki, and G. Anogianakis, "Cognitive event-related potentials: longitudinal changes in mild cognitive impairment," Clinical Neurophysiology, 2011. In press.

[65] C. L. Lai, R. T. Lin, L. M. Liou, and C. K. Liu, "The role of event-related potentials in cognitive decline in Alzheimer's disease," Clinical Neurophysiology, vol. 121, no. 2, pp. 194-199, 2010.

[66] T. Patel, R. Polikar, C. Davatzikos, and C. M. Clark, "EEG and MRI data fusion for early diagnosis of alzheimer's disease," in Proceedings of the 30th Annual International Conference of the IEEE Engineering in Medicine and Biology Society (EMBS '08), pp. 1757-1760, can, August 2008.

[67] M. Pierantozzi, M. Panella, M. G. Palmieri et al., "Different TMS patterns of intracortical inhibition in early onset Alzheimer dementia and frontotemporal dementia," Clinical Neurophysiology, vol. 115, no. 10, pp. 2410-2418, 2004.

[68] G. Pennisi, G. Alagona, R. Ferri et al., "Motor cortex excitability in Alzheimer disease: one year follow-up study," Neuroscience Letters, vol. 329, no. 3, pp. 293-296, 2002.

[69] P. Julkunen, A. M. Jauhiainen, S. Westerén-Punnonen et al., "Navigated TMS combined with EEG in mild cognitive impairment and Alzheimer's disease: a pilot study," Journal of Neuroscience Methods, vol. 172, no. 2, pp. 270-276, 2008.

[70] R. Polikar, C. Tilley, B. Hillis, and C. M. Clark, "Multimodal EEG, MRI and PET data fusion for Alzheimer's disease diagnosis," in Proceedings of the Annual International Conference of the IEEE Engineering in Medicine and Biology Society, vol. 1, pp. 6058-6061, 2010.

[71] M. van Gils, J. Koikkalainen, J. Mattila, S. Herukka, J. Lotjonen, and H. Soininen, "Discovery and use of efficient biomarkers for objective disease state assessment in alzheimer's disease," in Proceedings of the Annual International Conference of the IEEE Engineering in Medicine and Biology Society, vol. 1, pp. 2886-2889, 2010.

[72] A. Morinaga, K. Ono, T. Ikeda et al., "A comparison of the diagnostic sensitivity of MRI, CBF-SPECT, FDG-PET and cerebrospinal fluid biomarkers for detecting Alzheimer's disease in a memory clinic," Dementia and Geriatric Cognitive Disorders, vol. 30, no. 4, pp. 285-292, 2010.

[73] E. H. Corder, A. M. Saunders, N. J. Risch et al., "Protective effect of apolipoprotein E type 2 allele for late onset Alzheimer disease," Nature Genetics, vol. 7, no. 2, pp. 180-184, 1994.

[74] D. J. Berlau, K. Kahle-Wrobleski, E. Head, M. Goodus, R. Kim, and C. Kawas, "Dissociation of neuropathologic findings and cognition: case report of an apolipoprotein $\mathrm{E}$ epsilon2/epsilon2 genotype," Archives of Neurology, vol. 64, no. 8, pp. 1193-1196, 2007.

[75] G. Rodriguez, F. Nobili, G. Rocca, F. De Carli, M. V. Gianelli, and G. Rosadini, "Quantitative electroencephalography and regional cerebral blood flow: discriminant analysis between Alzheimer's patients and healthy controls," Dementia and Geriatric Cognitive Disorders, vol. 9, no. 5, pp. 274-283, 1998.

[76] G. Rodriguez, F. Nobili, F. Copello et al., "Tc-HMPAO regional cerebral blood flow and quantitative electroencephalography in Alzheimer's disease: a correlative study," Journal of Nuclear Medicine, vol. 40, no. 4, pp. 522-529, 1999.
[77] G. Rodriguez, F. Nobili, A. Arrigo et al., "Prognostic significance of quantitative electroencephalography in Alzheimer patients: preliminary observations," Electroencephalography and Clinical Neurophysiology, vol. 99, no. 2, pp. 123-128, 1996.

[78] F. Nobili, F. Copello, P. Vitali et al., "Timing of disease progression by quantitative EEG in Alzheimer's patients," Journal of Clinical Neurophysiology, vol. 16, no. 6, pp. 566-573, 1999.

[79] E. L. Helkala, V. Laulumaa, H. Soininen, J. Partanen, and P. J. Riekkinen, "Different patterns of cognitive decline related to normal or deteriorating EEG in a 3-year follow-up study of patients with Alzheimer's disease," Neurology, vol. 41, no. 4, pp. 528-532, 1991.

[80] J. J. Claus, B. W. Ongerboer De Visser, G. J. M. Walstra, A. Hijdra, B. Verbeeten Jr., and W. A. Van Gool, "Quantitative spectral electroencephalography in predicting survival in patients with early Alzheimer disease," Archives of Neurology, vol. 55, no. 8, pp. 1105-1111, 1998.

[81] O. L. Lopez, R. P. Brenner, J. T. Becker, R. F. Ulrich, F. Boller, and S. T. DeKosky, "EEG spectral abnormalities and psychosis as predictors of cognitive and functional decline in probable Alzheimer's disease," Neurology, vol. 48, no. 6, pp. 1521-1525, 1997.

[82] V. Jelic, P. Julin, M. Shigeta et al., "Apolipoprotein E $\varepsilon 4$ allele decreases functional connectivity in Alzheimer's disease as measured by EEG coherence," Journal of Neurology Neurosurgery and Psychiatry, vol. 63, no. 1, pp. 59-65, 1997.

[83] A. F. Leuchter, T. F. Newton, I. A. Cook, D. O. Walter, S. Rosenberg-Thompson, and P. A. Lachenbruch, "Changes in brain functional connectivity in Alzheimer-type and multiinfarct dementia," Brain, vol. 115, no. 5, pp. 1543-1561, 1992.

[84] T. Locatelli, M. Cursi, D. Liberati, M. Franceschi, and G. Comi, "EEG coherence in Alzheimer's disease," Electroencephalography and Clinical Neurophysiology, vol. 106, no. 3, pp. 229-237, 1998.

[85] Y. Wada, Y. Nanbu, Y. Koshino, N. Yamaguchi, and T. Hashimoto, "Reduced interhemispheric EEG coherence in Alzheimer disease: analysis during rest and photic stimulation," Alzheimer Disease and Associated Disorders, vol. 12, no. 3, pp. 175-181, 1998.

[86] V. Jelic, M. Blomberg, T. Dierks et al., "EEG slowing and cerebrospinal fluid tau levels in patients with cognitive decline," NeuroReport, vol. 9, no. 1, pp. 157-160, 1998.

[87] M. Lehtovirta, J. Partanen, M. Könönen et al., "A longitudinal quantitative EEG study of Alzheimer's disease: relation to apolipoprotein E polymorphism," Dementia and Geriatric Cognitive Disorders, vol. 11, no. 1, pp. 29-35, 2000. 


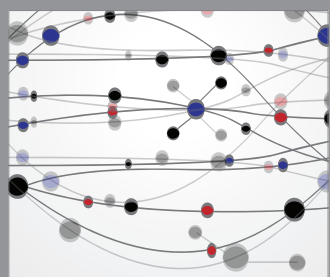

The Scientific World Journal
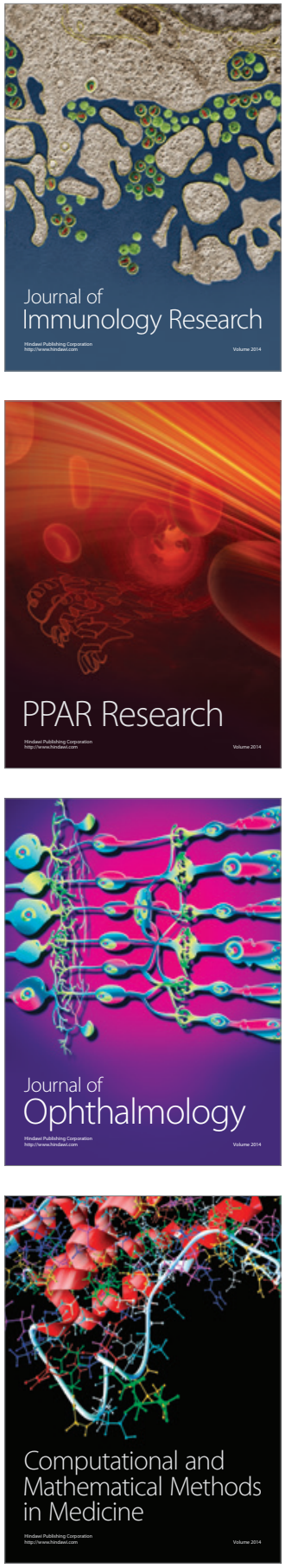

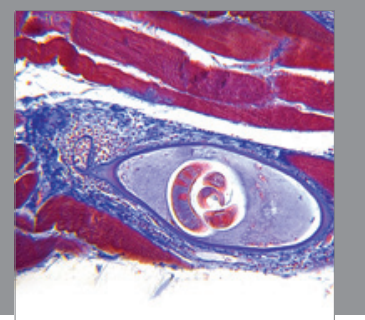

Gastroenterology

Research and Practice
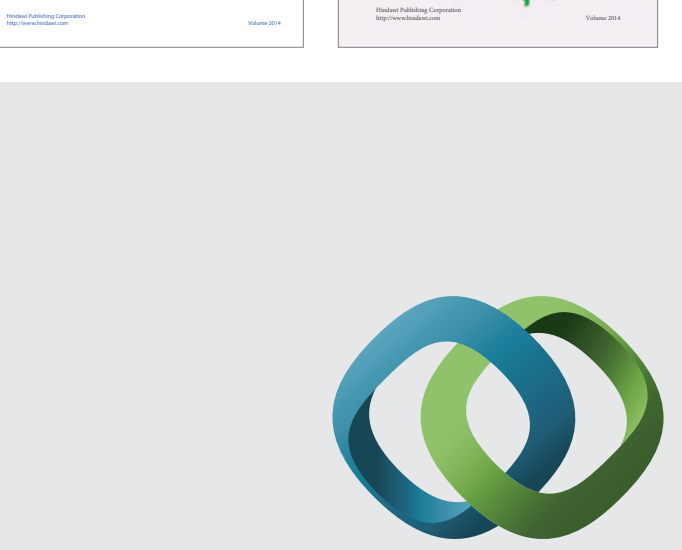

\section{Hindawi}

Submit your manuscripts at

http://www.hindawi.com
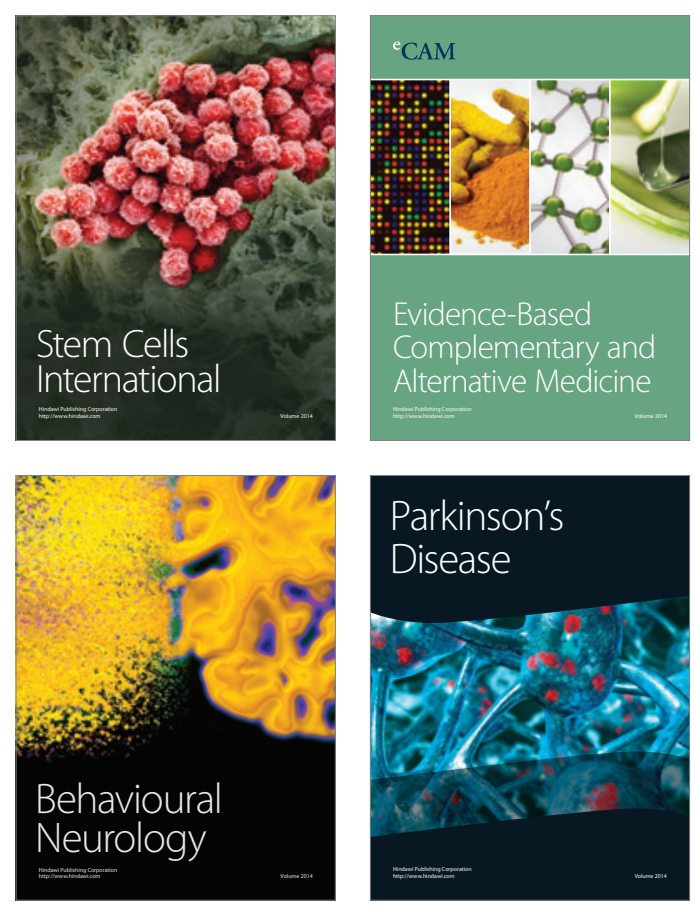

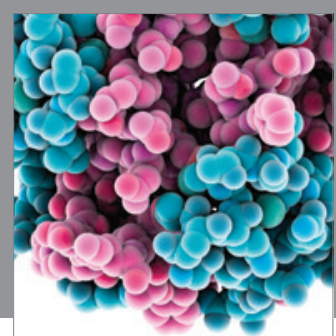

Journal of
Diabetes Research

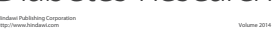

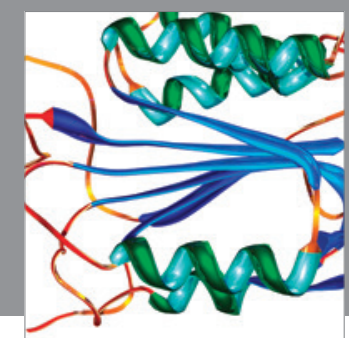

Disease Markers
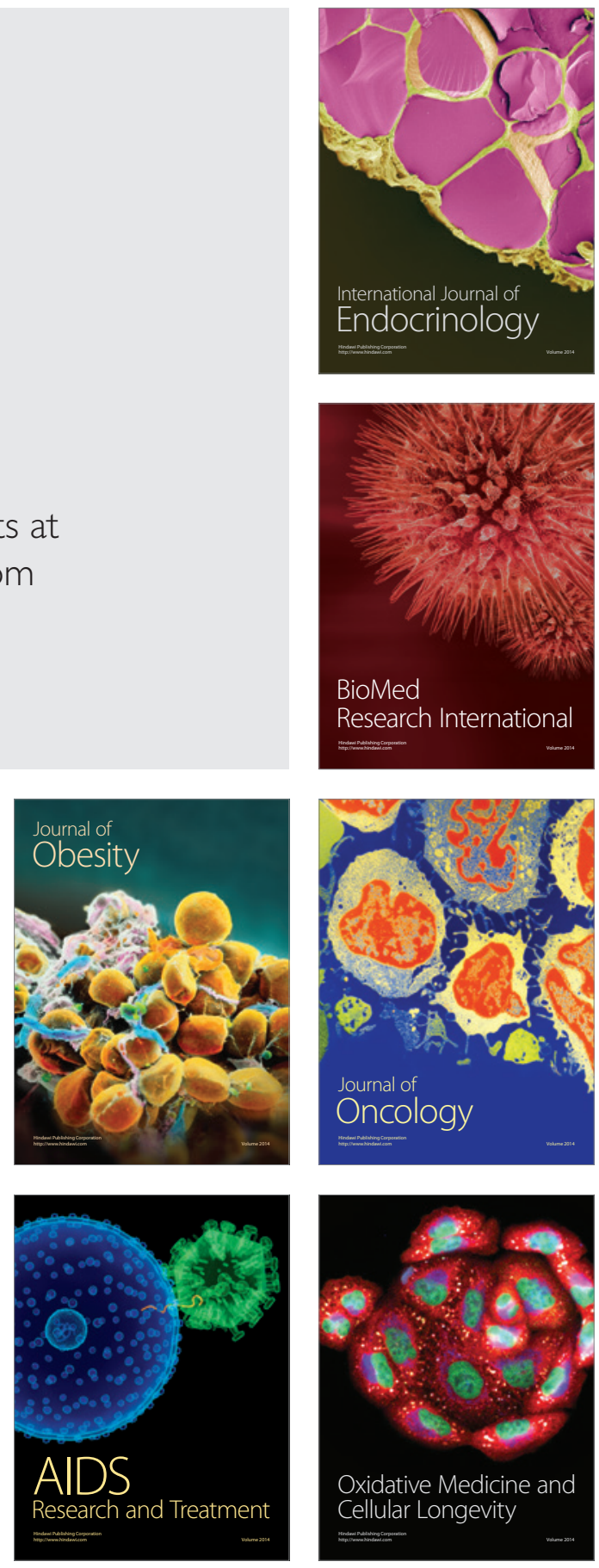